\title{
Some exact solutions for Toda type lattice differential equations using the improved (G'/G)-expansion method
}

\author{
İsmail Aslan ${ }^{* \dagger}$
}

\begin{abstract}
Nonlinear lattice differential equations (also known as differential-difference equations) appear in many applications. They can be thought of as hybrid systems for the inclusion of both discrete and continuous variables. On the basis of an improved version of the basic ( $\left.G^{\prime} / G\right)$-expansion method, we focus our attention towards some Toda type lattice differential systems for constructing further exact traveling wave solutions. Our method provides not only solitary and periodic wave profiles but also rational solutions with more arbitrary parameters. Copyright $\odot 2012$ John Wiley \& Sons, Ltd.
\end{abstract}

Keywords: $\quad\left(G^{\prime} / G\right)$-expansion method; lattice differential equation; differential-difference equation; traveling wave solutions

\section{Introduction}

Nonlinear lattice differential equations (NLDEs) are used in physical/mathematical models of a huge range of real-world phenomena. They occur, for instance, in biophysics, condensed matter physics, mechanical engineering, and in such fields as currents in electrical networks, molecular crystals, atomic chains, etc. Hence, their crucial role has motivated physicists, mathematicians, and other scientists to develop many integrable NLDEs, for example, see [1]. Contrary to difference equations, which are being fully discretized, NLDEs are semi-discretized with some (or all) of their space variables discretized while time is usually kept continuous. One of the goals in nonlinear science is to develop more general techniques that can be applied to a far wider variety of nonlinear equations. Recently, a start has been made on showing how methods of solution for nonlinear partial differential equations (NPDEs) can be adapted for application to NLDEs. In this direction, many well-known analytic methods initially developed for solving NPDEs have been successfully extended to a considerable number of NLDEs [2-5] even though there are some difficulties that usually arise when searching for iterative relations from indices $n$ to $n \pm i$. However, no method can provide all solutions to all types of NLDEs.

On the other hand, because of high-speed computing facilities, problems which in the past would have been intractable are now easily approximated by a number of basic computational techniques. In the literature, some modern analytic methods are available for dealing with NPDEs. Recently, Wang et al. [6] introduced the basic $\left(G^{\prime} / G\right)$-expansion method to find traveling wave solutions of NPDEs in mathematical physics. The method was very well received and a number of studies refining the initial idea appeared in the research literature [7-14]. The basis of the basic ( $\left.G^{\prime} / G\right)$-expansion method lies in the fact that it takes full advantage of linear theory. Contrary to the numerical computation of exact solutions of NLDEs, our algorithm computes closed-form analytical solutions that are polynomial in $\left(G^{\prime} / G\right)$. The solution procedure of the basic $\left(G^{\prime} / G\right)$-expansion method is easy, reliable, and efficient with the aid of a computer algebra system. If implemented in a rigorous manner, three types of solutions with arbitrary parameters can be constructed for NPDEs; hyperbolic, trigonometric, and rational. Naturally, the method has been extended, generalized and adapted by others [15-17] for various kinds of nonlinear problems. It is also remarkable that the generalization presented in [18] can be considered as a new step in studying NPDEs, see [19-24] for further applications. The present paper is a worthwhile contribution to this effort as well. We think that the application of new methods to the NLDEs with distinct structures is still an interesting and important problem of applied sciences.

Our objective in this study is twofold: first, to stress the power of the basic $\left(G^{\prime} / G\right)$-expansion method in handling NLDEs so that one can apply it for solving various types of nonlinear problems. Second, to extend it to two important Toda type lattice differential equations for the first time. The rest of this paper is organized as follows. In Section 2, we outline the improved (G'/G)-expansion method for the computation of closed-form solutions to NLDEs. In Section 3, we analyze our problems using the key steps of the method. Finally, some concluding remarks are given in Section 4. 


\section{The improved (G'/G)-expansion method for NLDEs}

Let us consider a system of $M$ polynomial NLDEs in the form

$$
\Delta\left(\mathbf{u}_{\mathbf{n}+\mathbf{p}_{1}}(\mathbf{x}), \ldots, \mathbf{u}_{\mathbf{n}+\mathbf{p}_{k}}(\mathbf{x}), \ldots, \mathbf{u}_{\mathbf{n}+\mathbf{p}_{1}}^{\prime}(\mathbf{x}), \ldots, \mathbf{u}_{\mathbf{n}+\mathbf{p}_{k}}^{\prime}(\mathbf{x}), \ldots, \mathbf{u}_{\mathbf{n}+\mathbf{p}_{1}}^{(r)}(\mathbf{x}), \ldots, \mathbf{u}_{\mathbf{n}+\mathbf{p}_{k}}^{(r)}(\mathbf{x})\right)=0,
$$

in which the dependent variable $\mathbf{u}_{\mathbf{n}}$ have $M$ components $u_{i, \mathbf{n}}$ and so do its shifts, the continuous variable $\mathbf{x}$ has $N$ components $x_{i}$, the discrete variable $\mathbf{n}$ has $Q$ components $n_{j}, \mathbf{p}_{s}(s=1, \ldots, k)$ denotes shift vectors $Q$ with components, and $\mathbf{u}^{(r)}(\mathbf{x})$ denotes the collection of mixed derivative terms of order $r$. To find traveling wave solutions of Equation (1), we first make the wave transformation

$$
\mathbf{u}_{\mathbf{n}+\mathbf{p}_{s}}(\mathbf{x})=\mathbf{U}_{\mathbf{n}+\mathbf{p}_{s}}\left(\xi_{\mathbf{n}}\right), \xi_{\mathbf{n}}=\sum_{i=1}^{Q} d_{i} n_{i}+\sum_{j=1}^{N} c_{j} x_{j}+\zeta_{,}(s=1,2, \ldots, k)
$$

where the coefficients $c_{1}, c_{2}, \ldots, c_{N}, d_{1}, d_{2}, \ldots, d_{Q}$ and the phase $\zeta$ are all constants. Then, Equation (1) becomes

$$
\Delta\left(\mathbf{U}_{\mathbf{n}+\mathbf{p}_{1}}\left(\xi_{\mathbf{n}}\right), \ldots, \mathbf{U}_{\mathbf{n}+\mathbf{p}_{k}}\left(\xi_{\mathbf{n}}\right), \ldots, \mathbf{U}_{\mathbf{n}+\mathbf{p}_{1}}^{\prime}\left(\xi_{\mathbf{n}}\right), \ldots, \mathbf{U}_{\mathbf{n}+\mathbf{p}_{k}}^{\prime}\left(\xi_{\mathbf{n}}\right), \ldots, \mathbf{U}_{\mathbf{n}+\mathbf{p}_{1}}^{(r)}\left(\xi_{\mathbf{n}}\right), \ldots, \mathbf{U}_{\mathbf{n}+\mathbf{p}_{k}}^{(r)}\left(\xi_{\mathbf{n}}\right)\right)=0
$$

We suppose that the solution of Equation (3) is in the finite series expansion form

$$
\mathbf{U}_{\mathbf{n}}\left(\xi_{\mathbf{n}}\right)=\sum_{l=-m}^{m} a_{l}\left(\frac{G^{\prime}\left(\xi_{\mathbf{n}}\right)}{G\left(\xi_{\mathbf{n}}\right)}\right)^{\prime}, a_{-m}^{2}+a_{m}^{2} \neq 0
$$

where $m$ is a positive integer to be specified, $a_{\text {I's }}$ are constants to be determined, $G\left(\xi_{\mathbf{n}}\right)$ satisfies the linear equation

$$
G^{\prime \prime}\left(\xi_{\mathbf{n}}\right)+\mu G\left(\xi_{\mathbf{n}}\right)=0,
$$

in which $\mu$ is an arbitrary constant to be determined, and the primes denote derivatives with respect to $\xi \mathbf{n}$. From the linear theory, the general solution of Equation (5) is well known to us. Thus, we have the following cases:

$$
\begin{aligned}
& \frac{G^{\prime}\left(\xi_{\mathbf{n}}\right)}{G\left(\xi_{\mathbf{n}}\right)}=\sqrt{-\mu}\left(\frac{C_{1} \cosh \left(\sqrt{-\mu} \xi_{\mathbf{n}}\right)+C_{2} \sinh \left(\sqrt{-\mu} \xi_{\mathbf{n}}\right)}{C_{1} \sinh \left(\sqrt{-\mu} \xi_{\mathbf{n}}\right)+C_{2} \cosh \left(\sqrt{-\mu} \xi_{\mathbf{n}}\right)}\right), \mu<0, \\
& \frac{G^{\prime}\left(\xi_{\mathbf{n}}\right)}{G\left(\xi_{\mathbf{n}}\right)}=\sqrt{\mu}\left(\frac{-C_{1} \sin \left(\sqrt{\mu} \xi_{\mathbf{n}}\right)+C_{2} \cos \left(\sqrt{\mu} \xi_{\mathbf{n}}\right)}{C_{1} \cos \left(\sqrt{\mu} \xi_{\mathbf{n}}\right)+C_{2} \sin \left(\sqrt{\mu} \xi_{\mathbf{n}}\right)}\right), \mu>0, \\
& \frac{G^{\prime}\left(\xi_{\mathbf{n}}\right)}{G\left(\xi_{\mathbf{n}}\right)}=\frac{C_{1}}{C_{1} \xi_{\mathbf{n}}+C_{2}}, \mu=0,
\end{aligned}
$$

where $C_{1}$ and $C_{2}$ are arbitrary constants. By a simple calculation, we can get the identity

$$
\xi \mathbf{n}+\mathbf{p}_{s}=\xi_{\mathbf{n}}+\varphi_{s}, \varphi_{s}=p_{s 1} d_{1}+p_{s 2} d_{2}+\cdots+p_{s Q} d_{Q}
$$

where $p_{s j}$ is the $j$ th component of the shift vector $\mathbf{p}_{s}$. Thus, considering the trigonometric/hyperbolic function identities and using the expressions (6a)-(6c) along with (7), we derive the shift functions as

$$
\begin{aligned}
& \mathbf{U}_{\mathbf{n}+\mathbf{p}_{s}}\left(\xi_{\mathbf{n}}\right)=\sum_{l=-m}^{m} a_{l}\left(\frac{\sqrt{-\mu} G^{\prime}\left(\xi_{\mathbf{n}}\right)-\mu \tanh \left(\sqrt{-\mu} \varphi_{s}\right) G\left(\xi_{\mathbf{n}}\right)}{\sqrt{-\mu} G\left(\xi_{\mathbf{n}}\right)+\tanh \left(\sqrt{-\mu} \varphi_{s}\right) G^{\prime}\left(\xi_{\mathbf{n}}\right)}\right)^{\prime}, a_{-m}^{2}+a_{m}^{2} \neq 0, \mu<0, \\
& \mathbf{U}_{\mathbf{n}+\mathbf{p}_{s}}\left(\xi_{\mathbf{n}}\right)=\sum_{l=-m}^{m} a_{l}\left(\frac{\sqrt{\mu} G^{\prime}\left(\xi_{\mathbf{n}}\right)-\mu \tan \left(\sqrt{\mu} \varphi_{s}\right) G\left(\xi_{\mathbf{n}}\right)}{\sqrt{\mu} G\left(\xi_{\mathbf{n}}\right)+\tan \left(\sqrt{\mu} \varphi_{s}\right) G^{\prime}\left(\xi_{\mathbf{n}}\right)}\right)^{\prime}, a_{-m}^{2}+a_{m}^{2} \neq 0, \mu>0, \\
& \mathbf{U}_{\mathbf{n}+\mathbf{p}_{s}}\left(\xi_{\mathbf{n}}\right)=\sum_{l=-m}^{m} a_{l}\left(\frac{G^{\prime}\left(\xi_{\mathbf{n}}\right)}{G\left(\xi_{\mathbf{n}}\right)+\varphi_{s} G^{\prime}\left(\xi_{\mathbf{n}}\right)}\right)^{\prime}, a_{-m}^{2}+a_{m}^{2} \neq 0, \mu=0 .
\end{aligned}
$$

According to the ansatz (4), we define the degree of $\mathbf{U}_{\mathbf{n}}\left(\xi_{\mathbf{n}}\right)$ as $D\left[\mathbf{U}_{\mathbf{n}}\left(\xi_{\mathbf{n}}\right)\right]=m$, which gives rise to the degree of other expressions as

$$
D\left[\mathbf{U}_{\mathbf{n}}^{(r)}\left(\xi_{\mathbf{n}}\right)\right]=m+r, D\left[\left(\mathbf{U}_{\mathbf{n}}^{(r)}\left(\xi_{\mathbf{n}}\right)\right)^{\beta}\right]=\beta(m+r), D\left[\left(\mathbf{U}_{\mathbf{n}}\left(\xi_{\mathbf{n}}\right)\right)^{\alpha}\left(\mathbf{U}_{\mathbf{n}}^{(r)}\left(\xi_{\mathbf{n}}\right)\right)^{\beta}\right]=\alpha m+\beta(m+r) .
$$


Balancing the highest order nonlinear term(s) and the highest-order derivative term in $\mathbf{U}_{\mathbf{n}}\left(\xi_{\mathbf{n}}\right)$ as in the continuous case, we can

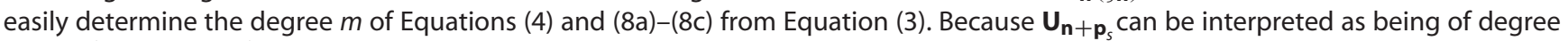
zero in $\left(G^{\prime}\left(\xi_{\mathbf{n}}\right) / G\left(\xi_{\mathbf{n}}\right)\right)$, the leading terms of $\mathbf{U}_{\mathbf{n}+\mathbf{p}_{s}}\left(\mathbf{p}_{s} \neq 0\right)$ will not affect the balancing procedure. Substituting the ansatz (4) and (8a)(8c) along with (5) into Equation (3), equating the coefficients of $\left(G^{\prime}\left(\xi_{\mathbf{n}}\right) / G\left(\xi_{\mathbf{n}}\right)\right)^{\prime}(I=0,1,2, \ldots)$ to zero, we obtain a system of nonlinear algebraic equations from which the undetermined constants $a_{l}, d_{i}, c_{j}$, and $\mu$ can be explicitly verified. Substituting these results into (4), we can derive various kinds of exact discrete solutions to Equation (1).

\section{Applications}

Among the various important aspects of lattice differential equations is the phenomenon of traveling wave solutions. In this section, we study two special types of Toda type lattice differential equations that have found a considerable amount of interest and attracted many researchers.

\subsection{The Toda lattice differential equation}

A famous model for discrete solutions is the integrable Toda lattice differential equation, which reads

$$
\frac{\mathrm{d}^{2} u_{n}}{\mathrm{~d} t^{2}}=\left(1+\frac{\mathrm{d} u_{n}}{\mathrm{~d} t}\right)\left(u_{n-1}(t)-2 u_{n}(t)+u_{n+1}(t)\right)
$$

where $u_{n}(t)=u(n, t)$ is the displacement of the $n$th particle from the equilibrium position. In this case, $\mathbf{u}=u, \mathbf{n}=n_{1}=n, \mathbf{x}=x_{1}=t$, and $\mathbf{p}_{1}=p_{1}=-1, \mathbf{p}_{2}=p_{2}=0, \mathbf{p}_{3}=p_{3}=1, d_{1}=d, c_{1}=c$. Now, for solving Equation (9), we first introduce the traveling wave transformation

$$
u_{n}=U_{n}\left(\xi_{n}\right), \xi_{n}=d n+c t+\zeta,
$$

where $d, c$ are constants to be determined later, and $\zeta$ is an arbitrary phase constant. Then, Equation (9) can be converted into

$$
c^{2} \frac{d^{2} U_{n}\left(\xi_{n}\right)}{d \xi_{n}^{2}}=\left(1+c \frac{d U_{n}\left(\xi_{n}\right)}{d \xi_{n}}\right)\left(U_{n-1}\left(\xi_{n}\right)-2 U_{n}\left(\xi_{n}\right)+U_{n+1}\left(\xi_{n}\right)\right) .
$$

We expand the solution of Equation (11) in the frame (4). Balancing the linear term of the highest order with the highest nonlinear term in (11) leads to $m=1$. Thus, we consider the ansatz

$$
U_{n}\left(\xi_{n}\right)=a_{0}+a_{1}\left(\frac{G^{\prime}\left(\xi_{n}\right)}{G\left(\xi_{n}\right)}\right)+a_{-1}\left(\frac{G^{\prime}\left(\xi_{n}\right)}{G\left(\xi_{n}\right)}\right)^{-1}, a_{-1}^{2}+a_{1}^{2} \neq 0,
$$

along with (5) for the discrete traveling wave solutions of Equation (9).

Case 1

When $\mu<0$, from (8a), we have

$$
U_{n \pm 1}\left(\xi_{n}\right)=\sum_{l=-1}^{1} a_{l}\left(\frac{\sqrt{-\mu} G^{\prime}\left(\xi_{n}\right) \mp \mu \tanh (\sqrt{-\mu} d) G\left(\xi_{n}\right)}{\sqrt{-\mu} G\left(\xi_{n}\right) \pm \tanh (\sqrt{-\mu} d) G^{\prime}\left(\xi_{n}\right)}\right)^{l}, a_{-1}^{2}+a_{1}^{2} \neq 0 .
$$

Substituting (12) and (13) along with (5) into (11), clearing the denominator and setting the coefficients of like powers of $\left(G^{\prime}\left(\xi_{n}\right) / G\left(\xi_{n}\right)\right)^{\prime}(0 \leq I \leq 10)$ to zero, we derive a system of nonlinear algebraic equations for $a_{0}, a_{1}, a_{-1}, d, c$, and $\mu$. Solving the resultant system (we omit to display them for simplicity) simultaneously, we get the following solution sets and the corresponding discrete hyperbolic function solutions of Equation (9):

Case 1.1

$$
\begin{gathered}
a_{0}=a_{0}, a_{-1}=0, a_{1}=\mp \frac{1}{\sqrt{-\mu}} \sinh (\sqrt{-\mu} d), c=\mp \frac{1}{\sqrt{-\mu}} \sinh (\sqrt{-\mu} d), d=d, \mu=\mu, \\
u_{n, 1}^{ \pm}(t)=a_{0} \mp \sinh (\sqrt{-\mu} d)\left(\frac{C_{1} \cosh \left(\sqrt{-\mu} \xi_{n}\right)+C_{2} \sinh \left(\sqrt{-\mu} \xi_{n}\right)}{C_{1} \sinh \left(\sqrt{-\mu} \xi_{n}\right)+C_{2} \cosh \left(\sqrt{-\mu} \xi_{n}\right)}\right),
\end{gathered}
$$

where $\xi_{n}=d n \mp \sinh (\sqrt{-\mu} d)(1 / \sqrt{-\mu}) t+\zeta$ and $\mu(<0), a_{0}, d, \zeta, C_{1}, C_{2}$ are arbitrary constants. Here and henceforth, the signs $( \pm)$ or (干) are ordered vertically. 
Case 1.2

$$
\begin{gathered}
a_{0}=a_{0,} a_{-1}= \pm \sqrt{-\mu} \sinh (\sqrt{-\mu} d), a_{1}=0, c= \pm \frac{1}{\sqrt{-\mu}} \sinh (\sqrt{-\mu} d), d=d, \mu=\mu, \\
u_{n, 2}^{ \pm}(t)=a_{0} \pm \sinh (\sqrt{-\mu} d)\left(\frac{C_{1} \cosh \left(\sqrt{-\mu} \xi_{n}\right)+C_{2} \sinh \left(\sqrt{-\mu} \xi_{n}\right)}{C_{1} \sinh \left(\sqrt{-\mu} \xi_{n}\right)+C_{2} \cosh \left(\sqrt{-\mu} \xi_{n}\right)}\right)^{-1},
\end{gathered}
$$

where $\xi_{n}=d n \pm \sinh (\sqrt{-\mu} d)(1 / \sqrt{-\mu}) t+\zeta$ and $\mu(<0), a_{0}, d, \zeta, C_{1}, C_{2}$ are arbitrary constants.

The expressions (15) and (17) represent abundant traveling wave solutions to Equation (9). Assigning special values to the parameters involved, we can construct formal solitary waves. For instance, if we let ' $C_{1}=0$ and $C_{2} \neq 0$ ' or ' $C_{1} \neq 0$ and $C_{2}=0$ ' in (15) then we get, respectively

$$
\begin{aligned}
& u_{n, 3}^{ \pm}(t)=a_{0} \mp \sinh (\sqrt{-\mu} d) \tanh (\sqrt{-\mu}(d n \mp \sinh (\sqrt{-\mu} d)(1 / \sqrt{-\mu}) t+\zeta)), \\
& u_{n, 4}^{ \pm}(t)=a_{0} \mp \sinh (\sqrt{-\mu} d) \operatorname{coth}(\sqrt{-\mu}(d n \mp \sinh (\sqrt{-\mu} d)(1 / \sqrt{-\mu}) t+\zeta)),
\end{aligned}
$$

where $\mu(<0), a_{0}, d$, and $\zeta$ are arbitrary constants.

Remark 1

As a special example, when $d=d_{1} / \sqrt{-\mu}, \zeta=\delta / \sqrt{-\mu}$, and $a_{0}=a_{10}$, our solution (18) turns out to be the solitary wave solution of Baldwin et al. [25] as expressed in their Table III. However, our solution (19) does not appear in there.

Case 2

When $\mu>0$, from (8b), we have

$$
U_{n \pm 1}\left(\xi_{n}\right)=\sum_{l=-1}^{1} a_{l}\left(\frac{\sqrt{\mu} G^{\prime}\left(\xi_{n}\right) \mp \mu \tan (\sqrt{\mu} d) G\left(\xi_{n}\right)}{\sqrt{\mu} G\left(\xi_{n}\right) \pm \tan (\sqrt{\mu} d) G^{\prime}\left(\xi_{n}\right)}\right)^{\prime}, a_{-1}^{2}+a_{1}^{2} \neq 0
$$

Substituting (12) and (20) along with (5) into (11), clearing the denominator and setting the coefficients of like powers of $\left(G^{\prime}\left(\xi_{n}\right) / G\left(\xi_{n}\right)\right)^{\prime}(0 \leq I \leq 10)$ to zero, we derive a system of nonlinear algebraic equations for $a_{0}, a_{1}, a_{-1}, d, c$, and $\mu$. Solving the resultant system simultaneously, we get the following solution sets and the corresponding discrete trigonometric function solutions of Equation (9):

Case 2.1

$$
\begin{gathered}
a_{0}=a_{0}, a_{-1}=0, a_{1}=\mp \frac{1}{\sqrt{\mu}} \sin (\sqrt{\mu} d), c=\mp \frac{1}{\sqrt{\mu}} \sin (\sqrt{\mu} d), d=d, \mu=\mu, \\
u_{n, 5}^{ \pm}(t)=a_{0} \mp \sin (\sqrt{\mu} d)\left(\frac{C_{2} \cos \left(\sqrt{\mu} \xi_{n}\right)-C_{1} \sin \left(\sqrt{\mu} \xi_{n}\right)}{C_{1} \cos \left(\sqrt{\mu} \xi_{n}\right)+C_{2} \sin \left(\sqrt{\mu} \xi_{n}\right)}\right),
\end{gathered}
$$

where $\xi_{n}=d n \mp \sin (\sqrt{\mu} d)(1 / \sqrt{\mu}) t+\zeta$ and $\mu(>0), a_{0}, d, \zeta, C_{1}, C_{2}$ are arbitrary constants.

Case 2.2

$$
\begin{gathered}
a_{0}=a_{0, a_{-1}}=\mp \sqrt{\mu} \sin (\sqrt{\mu} d), a_{1}=0, c= \pm \frac{1}{\sqrt{\mu}} \sin (\sqrt{\mu} d), d=d, \mu=\mu, \\
u_{n, 6}^{ \pm}(t)=a_{0} \mp \sin (\sqrt{\mu} d)\left(\frac{C_{2} \cos \left(\sqrt{\mu} \xi_{n}\right)-C_{1} \sin \left(\sqrt{\mu} \xi_{n}\right)}{C_{1} \cos \left(\sqrt{\mu} \xi_{n}\right)+C_{2} \sin \left(\sqrt{\mu} \xi_{n}\right)}\right)^{-1},
\end{gathered}
$$

where $\xi_{n}=d n \pm \sin (\sqrt{\mu} d)(1 / \sqrt{\mu}) t+\zeta$ and $\mu(>0), a_{0}, d, \zeta, C_{1}, C_{2}$ are arbitrary constants.

From the expressions (22) and (24) we can construct formal periodic waves. For example, if we let ' $C_{1}=0$ and $C_{2} \neq 0$ ' or ' $C_{1} \neq 0$ and $C_{2}=0^{\prime}$ in (22) then we get, respectively

$$
u_{n, 7}^{ \pm}(t)=a_{0} \mp \sin (\sqrt{\mu} d) \cot (\sqrt{\mu}(d n \mp \sin (\sqrt{\mu} d)(1 / \sqrt{\mu}) t+\zeta))
$$




$$
u_{n, 8}^{ \pm}(t)=a_{0} \pm \sin (\sqrt{\mu} d) \tan (\sqrt{\mu}(d n \mp \sin (\sqrt{\mu} d)(1 / \sqrt{\mu}) t+\zeta))
$$

where $\mu(>0), a_{0}, d$, and $\zeta$ are arbitrary constants.

Case 3

When $\mu=0$, from (8c), we have

$$
U_{n \pm 1}\left(\xi_{n}\right)=\sum_{l=-1}^{1} a_{l}\left(\frac{G^{\prime}\left(\xi_{n}\right)}{G\left(\xi_{n}\right) \pm d G^{\prime}\left(\xi_{n}\right)}\right)^{l}, a_{-1}^{2}+a_{1}^{2} \neq 0
$$

Substituting (12) and (27) along with (5) into (11), clearing the denominator and setting the coefficients of like powers of $\left(G^{\prime}\left(\xi_{n}\right) / G\left(\xi_{n}\right)\right)^{\prime}(0 \leq I \leq 10)$ to zero, we derive a system of nonlinear algebraic equations for $a_{0}, a_{1}, a_{-1}, d$, and $c$. Solving the resultant system simultaneously, we get the following solution set and the corresponding discrete rational function solution of Equation (9):

$$
\begin{gathered}
a_{0}=a_{0}, a_{-1}=0, a_{1}=\mp d, c=\mp d, d=d, \\
u_{n, 9}^{\mp}(t)=a_{0} \mp \frac{d C_{1}}{C_{1}(d n \mp d t+\zeta)+C_{2}},
\end{gathered}
$$

where $a_{0}, d, \zeta, C_{1}$, and $C_{2}$ are arbitrary constants.

Remark 2

We point out that the trigonometric solutions (25) and (26), and the rational solution (29) are not presented in [25].

\subsection{The Toda lattice differential system}

Let us consider the Toda lattice differential system

$$
\left\{\begin{aligned}
\frac{d u_{n}}{d t} & =u_{n}\left(v_{n}-v_{n-1}\right) \\
\frac{d v_{n}}{d t} & =v_{n}\left(u_{n+1}-u_{n}\right)
\end{aligned}\right.
$$

To look for traveling wave solutions of Equation (30), we first let

$$
u_{n}=U_{n}\left(\xi_{n}\right), v_{n}=V_{n}\left(\xi_{n}\right), \quad \xi_{n}=d n+c t+\zeta_{1}
$$

where $c, d$, and $\zeta$ are constants to be determined later, $\zeta$ is an arbitrary phase constant. Then, the system (30) reduces to

$$
\left\{\begin{array}{l}
c U_{n}^{\prime}-U_{n}\left(V_{n}-V_{n-1}\right)=0 \\
c V_{n}^{\prime}-V_{n}\left(U_{n+1}-U_{n}\right)=0
\end{array}\right.
$$

where $U_{n}=U_{n}\left(\xi_{n}\right), V_{n}=V_{n}\left(\xi_{n}\right)$, and the primes denote derivatives with respect to $\xi_{n}$. Balancing the linear term of the highest order with the highest nonlinear term in (32) according to the ansatz (4) leads to $m=1$. Thus, we assume that the solutions of the system (32) can be expressed in the form

$$
\left\{\begin{array}{l}
U_{n}=a_{0}+a_{1}\left(\frac{G^{\prime}\left(\xi_{n}\right)}{G\left(\xi_{n}\right)}\right)+a_{-1}\left(\frac{G^{\prime}\left(\xi_{n}\right)}{G\left(\xi_{n}\right)}\right)^{-1}, a_{-1}^{2}+a_{1}^{2} \neq 0 \\
V_{n}=b_{0}+b_{1}\left(\frac{G^{\prime}\left(\xi_{n}\right)}{G\left(\xi_{n}\right)}\right)+b_{-1}\left(\frac{G^{\prime}\left(\xi_{n}\right)}{G\left(\xi_{n}\right)}\right)^{-1}, b_{-1}^{2}+b_{1}^{2} \neq 0
\end{array}\right.
$$

where $G\left(\xi_{\mathbf{n}}\right)$ satisfies Equation (5), and $a_{0}, a_{1}, a_{-1}, b_{0}, b_{1}, b_{-1}$ are constants to be determined. The procedure is similar to the scheme used in Section 3.1; hence, we will omit most of the details here.

Case 1

For $\mu<0$, we get hyperbolic function solutions to the system (30) as

$$
\begin{aligned}
& \left\{\begin{array}{l}
u_{n, 1}^{\mp}(t)=c \sqrt{-\mu}\left(w_{n}(t)-\operatorname{coth}(d \sqrt{-\mu})\right), \\
v_{n, 1}^{\mp}(t)=-c \sqrt{-\mu}\left(w_{n}(t)+\operatorname{coth}(d \sqrt{-\mu})\right),
\end{array}\right. \\
& \left\{\begin{array}{l}
u_{n, 2}^{\mp}(t)=c \sqrt{-\mu}\left(\frac{1}{w_{n}(t)}-\operatorname{coth}(d \sqrt{-\mu})\right), \\
v_{n, 2}^{\mp}(t)=-c \sqrt{-\mu}\left(\frac{1}{w_{n}(t)}+\operatorname{coth}(d \sqrt{-\mu})\right),
\end{array}\right.
\end{aligned}
$$




$$
\left\{\begin{array}{l}
u_{n, 3}^{\mp}(t)=c \sqrt{-\mu}\left(\frac{1}{w_{n}(t)}+w_{n}(t)-2 \operatorname{coth}(2 d \sqrt{-\mu})\right), \\
v_{n, 3}^{\mp}(t)=-c \sqrt{-\mu}\left(\frac{1}{w_{n}(t)}+w_{n}(t)+2 \operatorname{coth}(2 d \sqrt{-\mu})\right),
\end{array}\right.
$$

where

$$
w_{n}(t)=\frac{C_{1} \cosh (\sqrt{-\mu}(d n+c t+\zeta))+C_{2} \sinh (\sqrt{-\mu}(d n+c t+\zeta))}{C_{1} \sinh (\sqrt{-\mu}(d n+c t+\zeta))+C_{2} \cosh (\sqrt{-\mu}(d n+c t+\zeta))}
$$

in which $\mu(<0), d, c, \zeta, C_{1}, C_{2}$ are arbitrary constants.

Of course, we can construct formal solitary waves from (34)-(36) as well. For instance, if we let ' $C_{1}=0$ and $C_{2} \neq 0$ ' or ' $C_{1} \neq 0$ and $C_{2}=0^{\prime}$ in (34) then we get, respectively

$$
\begin{gathered}
\left\{\begin{array}{l}
u_{n, 4}^{\mp}(t)=c \sqrt{-\mu}(-\operatorname{coth}(d \sqrt{-\mu})+\tanh (\sqrt{-\mu}(d n+c t+\zeta))), \\
v_{n, 4}^{\mp}(t)=-c \sqrt{-\mu}(\operatorname{coth}(d \sqrt{-\mu})+\tanh (\sqrt{-\mu}(d n+c t+\zeta))),
\end{array}\right. \\
\left\{\begin{array}{l}
u_{n, 5}^{\mp}(t)=c \sqrt{-\mu}(-\operatorname{coth}(d \sqrt{-\mu})+\operatorname{coth}(\sqrt{-\mu}(d n+c t+\zeta))), \\
v_{n, 5}^{\mp}(t)=-c \sqrt{-\mu}(\operatorname{coth}(d \sqrt{-\mu})+\operatorname{coth}(\sqrt{-\mu}(d n+c t+\zeta))),
\end{array}\right.
\end{gathered}
$$

where $\mu(<0), d, c, \zeta$ are arbitrary constants.

\section{Remark 3}

It is clear that (38) is included in Table III in [25] while (39) does not appear in there.

Case 2

For $\mu>0$, we get trigonometric function solutions to the system (30) as

$$
\begin{gathered}
\left\{\begin{array}{l}
u_{n, 6}^{\mp}(t)=c \sqrt{\mu}\left(w_{n}(t)-\cot (d \sqrt{\mu})\right), \\
v_{n, 6}^{\mp}(t)=-c \sqrt{\mu}\left(w_{n}(t)+\cot (d \sqrt{\mu})\right),
\end{array}\right. \\
\left\{\begin{array}{l}
u_{n, 7}^{\mp}(t)=-c \sqrt{\mu}\left(\frac{1}{w_{n}(t)}+\cot (d \sqrt{\mu})\right), \\
v_{n, 7}^{\mp}(t)=c \sqrt{\mu}\left(\frac{1}{w_{n}(t)}-\cot (d \sqrt{\mu})\right),
\end{array}\right. \\
\left\{\begin{array}{l}
u_{n, 8}^{\mp}(t)=c \sqrt{\mu}\left(-\frac{1}{w_{n}(t)}+w_{n}(t)-2 \cot (2 d \sqrt{\mu})\right), \\
v_{n, 8}^{\mp}(t)=-c \sqrt{\mu}\left(-\frac{1}{w_{n}(t)}+w_{n}(t)+2 \cot (2 d \sqrt{\mu})\right),
\end{array}\right.
\end{gathered}
$$

where

$$
w_{n}(t)=\frac{C_{2} \cos (\sqrt{\mu}(d n+c t+\zeta))-C_{1} \sin (\sqrt{\mu}(d n+c t+\zeta))}{C_{1} \cos (\sqrt{\mu}(d n+c t+\zeta))+C_{2} \sin (\sqrt{\mu}(d n+c t+\zeta))}
$$

in which $\mu(>0), d, c, \zeta, c_{1}, C_{2}$ are arbitrary constants.

As was accomplished before, we can construct formal periodic waves from (40)-(42) also. For instance, letting ' $C_{1}=0$ and $C_{2} \neq 0$ ' or ${ }^{\prime} C_{1} \neq 0$ and $C_{2}=0$ ' in (40) respectively leads to

$$
\begin{gathered}
\left\{\begin{array}{l}
u_{n, 9}^{\mp}(t)=c \sqrt{\mu}(-\cot (d \sqrt{\mu})+\cot (\sqrt{\mu}(d n+c t+\zeta))), \\
v_{n, 9}^{\mp}(t)=-c \sqrt{\mu}(\cot (d \sqrt{\mu})+\cot (\sqrt{\mu}(d n+c t+\zeta))),
\end{array}\right. \\
\left\{\begin{array}{l}
u_{n, 10}^{\mp}(t)=c \sqrt{\mu}(-\cot (d \sqrt{\mu})-\tan (\sqrt{\mu}(d n+c t+\zeta))), \\
v_{n, 10}^{\mp}(t)=-c \sqrt{\mu}(\cot (d \sqrt{\mu})-\tan (\sqrt{\mu}(d n+c t+\zeta))),
\end{array}\right.
\end{gathered}
$$

where $\mu(>0), d, c, \zeta$ are arbitrary constants.

Case 3

For $\mu=0$, we get a rational function solution to the system (30) as

$$
\left\{\begin{array}{l}
u_{n, 11}^{\mp}(t)=-\frac{c}{d}+\frac{c C_{1}}{c_{1}(d n+c t+\zeta)+C_{2}} \\
v_{n, 11}^{\mp}(t)=-\frac{c}{d}-\frac{c C_{1}}{C_{1}(d n+c t+\zeta)+C_{2}}
\end{array}\right.
$$

where $c, d, \zeta, C_{1}$, and $C_{2}$ are arbitrary constants. 
Remark 4

The triangular solutions (44) and (45) and the rational solution (46) are not derived in [25].

\section{Remark 5}

Compared with the standard procedure of the basic ( $\left.G^{\prime} / G\right)$-expansion method, our approach presents some improvements. The basic $\left(G^{\prime} / G\right)$-expansion method assumes an auxilary equation of the form $G^{\prime \prime}+\lambda G^{\prime}+\mu G=0$ where $\lambda$ and $\mu$ are arbitrary constants. Aslan [26] proved that one can take $\lambda=0$ without loss of generality. Taking (5) into account minimizes the number of involved parameters at the outset, which is clearly more advantageous. Our approach further simplifies the generalization of [18] in the sense that the sum goes from $I=-m$ to $I=m$ instead of from $I=0$ to $I=m$ in (4). Unlike the procedure of [18], we consider the case (8c) for obtaining rational solutions. The basic ( $\left.\mathrm{G}^{\prime} / \mathrm{G}\right)$-expansion method has also attracted some comments $[27,28]$, which depend on the problems studied.

\section{Conclusion}

We utilized a successful application of an improved version of the basic ( $\mathrm{G}^{\prime} / \mathrm{G}$ )-expansion method to two Toda type lattice differential equations. The method provides not only hyperbolic function solutions but also trigonometric and rational function solutions with more free parameters, which might imply some important physical meanings of the equations studied. Of course, the involved arbitrary parameters can be related to the initial/boundary conditions of the problem or can be set to other values resulting in different wave shapes. The rational solutions are obtained here for the first time and they could not be explicitly computed by other methods. We predict that our results can be useful for experts working on this field. Additional applications of the improved ( $\left.\mathrm{G}^{\prime} / \mathrm{G}\right)$-expansion method to other NLDEs deserve further investigation.

\section{References}

1. Toda M. Theory of nonlinear lattices. Springer: Berlin, 1989.

2. Zhu SD. Exp-function method for the hybrid-lattice system. International Journal of Nonlinear Sciences and Numerical Simulation 2007; 8(3):461-464.

3. Dai CQ, Meng JP, Zhang JF. Symbolic computation of extended Jacobian elliptic function algorithm for nonlinear differential-difference equations. Communications in Theoretical Physics 2005; 43(3):471-478.

4. Zhu SD, Chu YM, Qiu SL. The homotopy perturbation method for discontinued problems arising in nanotechnology. Computers \& Mathematics with Applications 2009; 58(11-12):2398-2401.

5. Yang P, Chen Y, Li ZB. ADM-Padé technique for the nonlinear lattice equations. Applied Mathematics and Computation 2009; 210(2):362-375.

6. Wang M, Li X, Zhang J. The ( $\left.G^{\prime} / G\right)$-expansion method and traveling wave solutions of nonlinear evolution equations in mathematical physics. Physics Letters A 2008; 372(4):417-423.

7. Zayed EME, Gepreel KA. Some applications of the ( $\left.\mathrm{G}^{\prime} / \mathrm{G}\right)$-expansion method to non-linear partial differential equations. Applied Mathematics and Computation 2009; 212(1):1-13.

8. Ganji DD, Abdollahzadeh M. Exact traveling solutions of some nonlinear evolution equation by (G'/G)-expansion method. Journal of Mathematical Physics 2009; 50(1):013519.

9. Aslan I, Öziş T. Analytic study on two nonlinear evolution equations by using the (G'/G)-expansion method. Applied Mathematics and Computation 2009; 209(2):425-429.

10. Aslan I, Öziş T. On the validity and reliability of the (G'/G)-expansion method by using higher-order nonlinear equations. Applied Mathematics and Computation 2009; 211(2):531-536.

11. Öziş T, Aslan I. Symbolic Computation and Construction of New Exact Traveling Wave Solutions to Fitzhugh-Nagumo and Klein-Gordon Equations. Zeitschrift für Naturforschung A 2009; 64a(1/2):15-20.

12. Öziş T, Aslan i. Symbolic computations and exact and explicit solutions of some nonlinear evolution equations in mathematical physics. Communications in Theoretical Physics 2009; 51(4):577-580.

13. Aslan I. Exact and explicit solutions to some nonlinear evolution equations by utilizing the (G'/G)-expansion method. Applied Mathematics and Computation 2009; 215(2):857-863.

14. Öziş T, Aslan I. Application of the (G'/G)-expansion method to Kawahara type equations using symbolic computation. Applied Mathematics and Computation 2010; 216(8):2360-2365.

15. Ebadi G, Biswas A. Application of the $\mathrm{G}^{\prime} / \mathrm{G}$-expansion method for nonlinear diffusion equations with nonlinear source. Journal of Franklin Intitute 2010; 347(7):1391-1398.

16. Zhang S, Tong JL, Wang W. A generalized (G'/G)-expansion method for the mKdV equation with variable coefficients. Physics Letters A 2008; 372(13):2254-2257.

17. Zayed EME, Al-Joudi S. Applications of an extended ( $\left.\mathrm{G}^{\prime} / \mathrm{G}\right)$-expansion method to find exact solutions of nonlinear PDEs in mathematical physics. Mathematical Problems in Engineering 2010; Art. ID 768573, 19.

18. Zhang S, Dong L, Ba J, Sun Y. The (G'/G)-expansion method for nonlinear differential-difference equations. Physics Letters A 2009; 373(10):905-910.

19. Aslan İ. Discrete exact solutions to some nonlinear differential-difference equations via the $\left(\mathrm{G}^{\prime} / \mathrm{G}\right)$-expansion method. Applied Mathematics and Computation 2009; 215(8):3140-3147.

20. Aslan I. The Ablowitz-Ladik lattice system by means of the extended (G'/G)-expansion method. Applied Mathematics and Computation 2009; 216(9):2778-2782.

21. Aslan I. Analytic solutions to nonlinear differential-difference equations by means of the extended ( $\left.G^{\prime} / G\right)$-expansion method. Journal of Physics $A$ : Mathematical and Theoretical 2010; 43(39):395207.

22. Aslan I. The extended discrete (G'/G)-expansion method and its application to the relativistic Toda lattice system. Numerical Analysis and Applied Mathematics, AIP Conference Proceedings 2009; 1:216-219.

23. Aslan i. The discrete $\left(G^{\prime} / G\right)$-expansion method applied to the differential-difference Burgers equation and the relativistic Toda lattice system. Numerical Methods for Partial Differential Equations. DOI: 10.1002/num.20611. 
24. Aslan i. A discrete generalization of the extended simplest equation method. Communications in Nonlinear Science and Numerical Simulation 2010; 15(8):1967-1973.

25. Baldwin D, Göktaş U, Hereman W. Symbolic computation of hyperbolic tangent solutions for nonlinear differential-difference equations. Computer Physics Communications 2004; 162(3):203-217.

26. Aslan I. A note on the (G'/G)-expansion method again. Applied Mathematics and Computation 2010; 217(2):937-938.

27. Parkes E. Observations on the basic $\left(\mathrm{G}^{\prime} / \mathrm{G}\right)$-expansion method for finding solutions to nonlinear evolution equations. Applied Mathematics and Computation 2010; 217(4):1759-17634.

28. Kudryashov N. A note on the (G'/G)-expansion method. Applied Mathematics and Computation 2010; 217(4):1755-1758. 